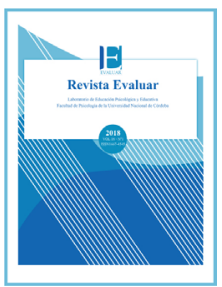

\title{
Propiedades métricas de la Escala Salvadoreña de Autoritarismo de Derechas (RWA)
}

\section{Metric properties of the Salvadorian Right-Wing Authoritarianism Scale (RWA)}

\author{
Carlos Iván Orellana * 1 \\ 1 - Universidad Don Bosco, El Salvador.
}

\author{
Introducción \\ Método \\ Resultados \\ Discusión \\ Referencias
}

Recibido: 03/10/2017 Revisado: 29/11/2017 Aceptado: 06/12/2017

\section{Resumen}

El objetivo de este trabajo es exponer las propiedades métricas de la Escala Salvadoreña de Autoritarismo de Derechas (RWA), desarrollada por Orellana (2017). Se contó con una muestra de 421 habitantes del Área Metropolitana de San Salvador (AMSS) mayores de 15 años $(\mathrm{M}=39.2$; $\mathrm{DE}=17.2)$. La escala es el producto de un proceso de depuración de preguntas adaptadas o incluidas en encuestas de opinión y de cultura política. De acuerdo con el coeficiente alfa de Cronbach, la escala presenta una consistencia interna total satisfactoria $(\alpha=.83)$. Un análisis factorial confirmatorio evidencia un modelo con un ajuste adecuado que reproduce la reconocida estructura factorial tridimensional del RWA de Altemeyer (1996; validez de constructo). La escala está correlacionada con constructos determinados ( $\mathrm{p}$. ej., dimensiones de cultura política, inseguridad ciudadana y disposiciones psicosociales como prejuicio) en concordancia teórica y empírica con la tradición de estudios sobre autoritarismo (validez de criterio-concurrente). Se discuten sus características y futuras vías de aplicación.

Palabras clave: autoritarismo de derechas, análisis factorial confirmatorio, validez, confiabilidad, índices de bondad de ajuste, correlaciones

\begin{abstract}
The aim of this work is to expose the metrical properties of the Salvadorian Right Wing Authoritarianism Scale (RWA), developed by Orellana (2017). Based on a sample of 421 inhabitants, 15 years of age and older $(\mathrm{M}=$ 39.2; $\mathrm{DE}=17.2$ ) from the San Salvador Metropolitan Area (SSMA), the scale is the product of a process of selection of questions already adapted or included in public opinion surveys or political culture polls. According to the Cronbach's Alpha coefficient, the scale presents a satisfactory total internal consistence $(\alpha=.83)$. A confirmatory factorial analysis evidences an adequately fitting model, that reproduces the recognized three-dimensional factorial structure of Altemeyer's RWA (1996; construct validity). The scale correlates with certain constructs (e.g., dimensions of political culture, citizen insecurity and psychosocial dispositions as prejudice) in theoretical and empirical concordance with the former studies on authoritarianism (concurrent-criterion validity). Its characteristics and future ways of application are discussed.
\end{abstract}

Keywords: right-wing authoritarianism, confirmatory factor analysis, validity, reliability, goodness of fit indexes, correlations 


\section{Introducción}

El Salvador es un país eufemísticamente considerado como de desarrollo medio que en el último cuarto de siglo ha pasado por transformaciones sociales y políticas considerables. En la primera mitad del decenio de 1990, y viniendo de décadas de gobiernos militares y de una guerra civil, el país asiste a la instauración de un régimen democrático y un modelo económico neoliberal. Paralelamente, presenta altos niveles de exclusión y desigualdad social, así como de violencia y criminalidad alimentadas por el fenómeno de las pandillas y su fallido combate estatal represivo (Instituto Universitario de Opinión Pública [IUDOP], 2014; Orellana, 2012; Programa de las Naciones Unidas para el Desarrollo [PNUD], 2013). En otras palabras, se trata de un contexto subdesarrollado que exhibe antecedentes institucionales autoritarios, una cultura conservadora y rudimentaria en términos democráticos, ineficiencia y punitividad institucional, precariedad vital y niveles epidémicos de inseguridad ciudadana y de violencia criminal enquistados profundamente en su tejido social.

Una de las consecuencias del proceso de democratización que coexiste con condiciones sociales y materiales adversas fue la proliferación de encuestas de opinión y de cultura política dirigidas a indagar dimensiones diversas de la vida nacional: preocupaciones cotidianas, aspectos de convivencia, victimización e inseguridad, valores democráticos, intenciones electorales, entre otros aspectos. En ellas, las orientaciones autoritarias fueron apareciendo de forma bastante diáfana. La preferencia por ley y orden antes que por derechos humanos o por la eliminación con mano dura de quienes causen problemas (IUDOP, 1998); el rechazo a convivir con personas homosexuales o a las uniones matrimoniales de personas del mismo sexo, la aprobación de limpiezas sociales y de la prevalencia masiva e intergeneracional del castigo físico en la infancia (IUDOP, 1999a, 1999b; Speizer, Goodwin, Samandari, Kim, \& Clyde, 2008) y la aprobación social de la implementación de la pena de muerte (Cruz, 1999), entre otras manifestaciones represivas y antidemocráticas.

Asimismo, los sondeos del Barómetro de las Américas muestran que, entre 2004 y 2014, los niveles de intolerancia política han incrementado al grado que en el país la combinación entre apoyo al sistema democrático y la tolerancia política ubicaría a casi siete de cada 10 personas en categorías de democracia en riesgo $(30.6 \%)$ y de estabilidad autoritaria (38.7\%; Córdova-Macías, Cruz, \& Zeichmeister, 2015). Cabe añadir que, como vienen mostrando distintas encuestas de victimización a lo largo de los años, en la actualidad, la gran mayoría de los habitantes de El Salvador $(83 \%)$ experimenta una inseguridad masiva y ubicua, "total" (Latinobarómetro, 2015). Por último, una investigación reciente (Cruz, Aguilar, \& Vorobyeva, 2017) encontró que un $40 \%$ de la población aprobaría que la policía torture a delincuentes para conseguir información sobre el crimen organizado.

Esto quiere decir que en El Salvador el autoritarismo emerge en distintos sondeos de opinión de forma incontestable. Pero se trata de indicios dispersos más centrados en el dato mismo que en la ratificación de un trasfondo teórico particular. Por ejemplo, en su momento los trabajos de Cruz (1999) y de Seligson, Cruz y Córdova-Macías (2000) aluden a la teoría del Autoritarismo de ala derecha o Right-Wing Authoritarinism (RWA) de Altemeyer (1996) pero no guardan especial fidelidad teórica al constructo con las mediciones realizadas. Esto cambiará con el desarrollo de un sondeo de opinión llevado a cabo con adultos jóvenes urbanos y en el marco propicio de un trabajo de postgrado (IUDOP, 2002), cuando se desarrolla una escala que sí refleja la estructura tridimensio- 
nal del RWA de Altemeyer (1981, 1996) (escala original de 41 ítems luego depurados a 38, $\alpha=$ .85 y tres factores que en conjunto explicaban el $29.9 \%$ de la varianza de los resultados). Sobre la versión reducida de la escala en cuestión versa este trabajo.

\section{La medida del autoritarismo}

La evolución histórica de la medición del autoritarismo en psicología social y psicología política se ha producido con el telón de fondo de las transformaciones teóricas propias del estudio del prejuicio (Molero, 2007). Según Duckitt (1992), el estudio del prejuicio habría transitado durante casi un siglo por teorías raciales, psicodinámicas, socioculturales y grupales hasta alcanzar una perspectiva cognitiva. En este avance teórico-metodológico rápidamente despuntó el empleo de instrumentos estandarizados para aproximarse al fenómeno. La clásica Escala $\mathrm{F}$ incluida en el emblemático estudio de la década de los 50 de Adorno, Frenkel-Brunswik, Levinson y Sanford (1969), la Escala de Dogmatismo de Rokeach (1960), la Escala de Autoritarismo de Derechas (Right-Wing Authoritarianism o RWA, por sus siglas en inglés) de Altemeyer (1981), la dinámica autoritaria de los valores de crianza de Stenner (2005) y debates y aproximaciones al autoritarismo de izquierda (Altemeyer, 1996; Ovejero, 1992; Stone, 1990; Van Hiel, Duriez, \& Kossowska, 2006) dan cuenta tanto de la persistencia como de la vigencia del esfuerzo por capturar empíricamente el autoritarismo.

Hoy en día se reconoce que, con sus detractores y sus defensores (Roccato \& Converso, 1996; Stenner, 2005), el RWA de Altemeyer $(1996,1998)$ constituye la propuesta más aceptada en términos teóricos y metodológicos para el estudio del autoritarismo como realidad sub- jetiva. El RWA se estructura a partir de tres dimensiones remanentes de la conocida Escala F: la agresión autoritaria o la manifestación de tendencias hostiles o punitivas; el convencionalismo, es decir, orientaciones tradicionales y de respeto por lo establecido y la sumisión autoritaria como tendencia a seguir los dictados de autoridades consideradas como legítimas (Altemeyer, 1996, 2006). El RWA se fundamenta en el aprendizaje social y se comprende como una actitud, como una expresión de intolerancia y punitividad antes que como una predisposición o una personalidad autoritaria (Altemeyer, 1996; Molero, 2007; Stenner, 2005).

Siguiendo a Duckitt (2001, 2003), el RWA sería el producto complejo de una socialización coercitiva, estructuraciones psicológicas conformistas y rígidas y la percepción de un mundo amenazante que deriva en animadversión hacia grupos considerados como no convencionales (p. ej., comunidad LGTBI). Precisamente uno de los aspectos más característicos en la historia del estudio del autoritarismo es el papel de las amenazas percibidas -el desarraigo del mundo, el quiebre de las buenas costumbres, la inseguridad debido al crimen, la amenaza del terrorismo, grupos objeto de prejuicio- como detonantes o moduladores fundamentales de estas tendencias antidemocráticas (Adorno et al., 1969; Duckitt, 2003; Feldman \& Stenner, 1997; Merolla \& Zechmeister, 2009; Seligson et al., 2000; Sibley, Wilson, \& Duckitt, 2007; Stenner, 2005).

Podría decirse que la medición del RWA ha seguido un proceso de estabilización, depuración $\mathrm{y}$ adaptación en las últimas cuatro décadas (A1temeyer, 1981, 1996, 1998, 2006). Mientras que con el pasar del tiempo la escala de medición del constructo se posiciona ante la comunidad académica como una medida válida de actitudes autoritarias y experimenta un proceso de variación, reformulación y acortamiento de ítems (Altemeyer, 2006), paralelamente proliferan esfuerzos de 
administración, innovación y de traducción de la escala en contextos diferentes al norteamericano angloparlante (Canetti \& Pedahzur, 2002; Giampaglia \& Roccato, 2002; Zakrisson, 2005), lo que incluye también países de habla hispana (Etchezahar, 2012; Moya \& Morales-Marente, 2005; Seoane \& Garzón, 1992).

Por lo apuntado, resulta claro que en El Salvador existen condiciones contextuales así como indicios empíricos inequívocos de la existencia de orientaciones autoritarias en la población. También resulta claro que se dispone de reconocidas alternativas de medición del fenómeno. No obstante, en el país no se han llevado a cabo aproximaciones empíricas con el concurso de escalas consolidadas para medir el autoritarismo. El objetivo de este trabajo es mostrar las características métricas (validez y confiabilidad) de una escala de actitudes autoritarias que permita contar con un instrumento unificado y teóricamente fundamentado para la medición del constructo en sociedades con características similares a las de El Salvador.

\section{Método}

\section{Participantes}

Se contó con una muestra representativa de los habitantes del Área Metropolitana de San Salvador (AMSS), la zona geográfica del país fundamentalmente urbana con el más alto pero heterogéneo desarrollo socioeconómico del país. Las características de la muestra correspondieron con los parámetros poblacionales establecidos en las proyecciones de población para el 2010 de la Dirección General de Estadística y Censos del Ministerio de Economía (DIGESTYC, 2009). En función de los recursos disponibles, y considerando que se contaba con una población infinita, se estableció el tamaño de la muestra a partir de los criterios siguientes: un $95 \%$ de confiabilidad (Z), una varianza del $50 \%$ (p) y un error muestral (E) inferior al $4.8 \%$.

El total de participantes fue de 421 personas mayores de 15 años $(\mathrm{M}=39.2$ años, $\mathrm{DE}=$ $17.2), 44.2 \%$ hombres y $55.8 \%$ mujeres. En cuanto al nivel educativo, $41.1 \%$ contaba con estudios máximos de noveno grado, 28\% había alcanzado el bachillerato y el restante $30.9 \%$ contaba con estudios técnicos o universitarios. Todos los participantes fueron elegidos al azar en concordancia con las proporciones y características de inclusión sociodemográfica de la población de origen.

\section{Instrumento}

La escala que aquí se describe responde a una construcción/adaptación de la escala RWA de Altemeyer $(1996,1998,2006)$ en los términos que se desprenden del debate expuesto por Fernández, Pérez, Alderete, Richaud y Fernández-Liporace (2010). Ahí se dice, siguiendo específicamente la postura de Richaud, que ante las diferencias de contexto de producción de las escalas de medición debe priorizarse la funcionalidad de las mismas antes que su literalidad. Además, se dice que la mera adaptación a través de simples traducciones es "desaconsejable", por lo que "lo más pertinente sería construir nuevas pruebas que operacionalicen exactamente el concepto, atributo o procesos que el investigador desea estudiar, dentro de su contexto teórico y cultural y siguiendo los principios psicométricos adecuados" ( $p$. 68).

La estructura de las distintas variantes de la escala RWA de Altemeyer (p. ej., Altemeyer, 2006) presentan opciones de respuesta complejas (números negativos, procedimientos aritméticos, etc.), ítems extensos y compuestos (Etchezahar, 2012) y expresiones cuya traducción precisa al 
español resulta dificultosa. Por ello se decidió desarrollar una escala según los términos expuestos por Fernández et al. (2010) que respondiera a la teoría tridimensional del RWA pero no a su forma, no a una mera traducción de alguna de las versiones disponibles de la escala. Interesaba tomar en cuenta las características promedio de la población salvadoreña que participa en los sondeos nacionales de opinión ( $\mathrm{p}$. ej., niveles educativos modestos, en medio de sus labores cotidianas) así como la experiencia acumulada sobre ciertos tecnicismos que deben cumplir las preguntas en este tipo de pesquisas (i.e., oraciones cortas y estructura de respuesta tipo Likert no superior a cinco puntos).

Para la construcción de la escala se retomaron ideas de algunos ítems de la escala de Altemeyer (1996), pero se ajustaron su extensión y su redacción al manejo del lenguaje y la realidad salvadoreña. Otros ítems fueron retomados de encuestas de cultura política realizadas por el IUDOP y, finalmente, el resto de los ítems fue redactado ad hoc con base en la teoría de las dimensiones del constructo RWA (Altemeyer, 1981, 1996). Esto explica que algunos ítems hagan alusión a aspectos muy específicos de la realidad salvadoreña como las pandillas o ciertas creencias religiosas. Las importantes variantes de forma y de contenido mencionadas como la muestra participante justifican el hablar de una Escala Salvadoreña de Autoritarismo de Derechas (RWA). Cabe aclarar que, en otra parte (Orellana, 2017), esta escala fue identificada como SRWA (Salvadoran Right-Wing Authoritarianism Scale) en alusión expresa a la deuda teórica con la reconocida escala RWA de Altemeyer, pero al mismo tiempo para enfatizar el proceso particular de construcción/ adaptación seguido así como para homologarla con las convenciones terminológicas vigentes para el estudio empírico del autoritarismo.

La premisa seguida para construir/adaptar la escala fue procurar consistencia interna pero también relevancia teórica y contextual de los ítems que finalmente constituirían las tres dimensiones del RWA (John \& Benet-Martínez, 2000), una vez se había asumido que la validez podía darse por descontada. La escala RWA finalmente quedó compuesta por 22 ítems: ocho corresponden a la dimensión de agresión autoritaria, nueve a la de convencionalismo y cinco a la de sumisión autoritaria. La estructura de respuesta de la escala es de tipo Likert de cinco opciones, a saber: (0) Muy en desacuerdo, (1) En desacuerdo, (2) Indeciso, (3) Algo de acuerdo y (4) Muy de acuerdo. Esta forma de respuesta y sus valores implican que a mayor puntaje y acuerdo con los ítems, mayor actitud autoritaria, mientras un menor puntaje y desacuerdo con las proposiciones, indicarían una actitud libertaria, según la crítica de Stenner (2005) dirigida a la interpretación imprecisa de Altemeyer $(1981,1996)$ de los puntajes bajos de la escala RWA. En el Apéndice 1 pueden apreciarse los ítems de la escala RWA separados por dimensiones, la consigna general de la escala y sus opciones de respuesta.

La escala salvadoreña RWA fue incluida en un cuestionario tipo encuesta conformado por un total de 117 preguntas, pero sus tres subescalas se presentaron de forma separada para evitar aquiescencia ya que todos están redactados en sentido positivo. En el cuestionario tipo encuesta, además de la escala de autoritarismo, fueron incluidas otras preguntas y otras escalas sobre temáticas diversas; entre ellas, preguntas categóricas genéricas de corte sociodemográfico (p. ej., sexo: masculino, femenino; religión: ninguna, católica, evangélica, otra). Otras preguntas se enfocaron en la evaluación de la situación del país: ítems en los que en una escala de tres puntos -(1) ha mejorado, (2) sigue igual, (3) ha empeorado- el entrevistado establece según su criterio si la delincuencia, la economía o las leyes para combatir 
la delincuencia han cambiado para mejor o para peor.

Asimismo, la encuesta incluyó un área de cultura política. Aquí se incluyeron aspectos como el autoposicionamiento ideológico del entrevistado en una escala de 10 puntos: (1) Izquierda-(10) Derecha. También se incluyó la preferencia por un régimen político de corte democrático o autoritario equivalente a una pregunta categórica enfocada en la legitimidad democrática (p. ej., Córdova-Macías et al., 2015). Adicionalmente, se contó con un área referida a inseguridad y victimización en la que se incluyen preguntas sobre las pandillas como mucho o nada de problema en la comunidad en una escala de cuatro puntos; preguntas categóricas concernientes a la portación o al deseo de hacerse con un arma de fuego y al hecho de haber visto o no una persona asesinada en la calle; se incluyó además una escala presentada como acciones precautorias en la cual el entrevistado seleccionaba en una escala dicotómica (sí o no) medidas tomadas debido a su preocupación por el crimen (p. ej., organización con vecinos, instalación de alarmas).

Entre las escalas más complejas incluidas en la encuesta, debido a su trasfondo teórico, se encuentran las escalas de prejuicio hacia los delincuentes, la de anomia y la de disposición autoritaria. La escala de prejuicio presenta una estructura de respuesta Likert de cuatro puntos, desde (0) Muy en desacuerdo hasta (4) Muy de acuerdo, y fue construida expresamente para la investigación. Reúne ítems sobre prejuicio manifiesto (p. ej., locura, naturaleza) y sutil (p. ej., diferencia de valores, de formas de crianza) hacia los delincuentes según la argumentación de Allport (1954). La escala de anomia constituye una fusión de las escalas clásicas de Srole (1956) y de McClosky y Schaar (1965) y alude a aspectos de desconcierto, desapego y desmoralización con la situación circundante. También se trató de una es- cala Likert, pero de cinco puntos: Muy de acuerdo, De acuerdo, Indeciso, En desacuerdo, Muy en desacuerdo. Por último, la escala de disposición autoritaria responde a tres pares de opciones (una escala dicotómica) sobre la preferencia por patrones de crianza libertarios (p. ej., que el niño piense por sí mismo) o verticalistas (p. ej., que el niño sea obediente a sus padres) que, según Stenner (2005), constatan disposiciones autoritarias básicas sin realizar medidas intrusivas o cargadas políticamente.

\section{Procedimiento}

El trabajo de campo que permitió la administración de la escala RWA fue realizado en 2010 siguiendo un muestreo que fue denominado como probabilístico estratificado a través de selección sistemática de elementos muestrales (Bordens \& Abott, 1999; Hernández-Sampieri, Fernández-Collado, \& Baptista Lucio, 2006). Es decir que se realizó una selección aleatoria de unidades muestrales repartidas en segmentos urbanos a partir de características de inclusión determinadas (p. ej., contar con 15 años o más). Se trata de un proceso polietápico que garantiza dispersión y representatividad muestral. El trabajo de campo fue desarrollado por encuestadores entrenados. Al inicio de la entrevista se aseguró el consentimiento informado y la confidencialidad de la información, así como la posibilidad de contacto ante cualquier inquietud surgida en el proceso de entrevista.

\section{Los análisis realizados}

Los análisis han sido llevados a cabo con el programa SPSS y AMOS v. 20 (Arbuckle, 2012). Los puntajes de la escala RWA y los de sus subes- 
calas o dimensiones fueron convertidos a rangos de 0 a 10 para facilitar su lectura siguiendo las sencillas pautas de conversión de puntajes sugeridas por Fournier y Garner (1990) donde cero indicaría el máximo de libertarismo y 10 el máximo de autoritarismo.

A continuación se presentan las estadísticas descriptivas y bivariadas que exponen resultados generales obtenidos por la muestra participante. Asimismo, la confiabilidad de la escala RWA y de sus tres dimensiones ha sido obtenida recurriendo al coeficiente alfa de Cronbach. Mientras que se asume la validez de contenido de la escala debido a que la misma ha sido el producto de un proceso sistemático de selección y de depuración de un universo comprehensivo de ítems sobre autoritarismo, aquí se presentan análisis correspondientes a la validez de constructo a través de un análisis factorial confirmatorio (AFC) realizado con el método de estimación de componentes principales y rotación varimax. La bondad de ajuste del modelo factorial será determinada por los índices de bondad de ajuste siguientes: $\chi 2 / \mathrm{gl}$, GFI, AGFI, CFI, RMR y RMSEA. Siguiendo la literatura (Byrne, 2010; Hooper, Coughlan, \& Mullen, 2008; Schreiber, Nora, Stage, Barlow, \& King, 2006), serán considerados como indicadores de buen ajuste los valores de $\chi 2 / \mathrm{gl}$ inferiores a 2.0, GFI y AGFI superiores a .90, CFI mayor o igual que .95 , RMR cercano a 0.0 y RMSEA inferior a .06. Por último, la validez de criterio concurrente será obtenida por medio de correlaciones bivariadas que resultan teóricamente coherentes entre el RWA y otros indicadores de cultura política, de inseguridad y de otras disposiciones psicosociales (Bordens \& Abott, 1999; Cubo-Delgado, Mar

Tabla 1

Medias y desviaciones de la escala RWA y sus dimensiones según variables sociodemográficas.

\begin{tabular}{|c|c|c|c|c|}
\hline & RWA & $\begin{array}{c}\text { Agresión } \\
\text { Autoritaria }\end{array}$ & Convencionalismo & Sumisión Autoritaria \\
\hline Variables & M(DE) & M(DE) & M(DE) & $\mathbf{M}(\mathbf{D E})$ \\
\hline TODOS/AS & $6.3(1.7)$ & $4.9(2.9)$ & $6.9(2.1)$ & $5.5(1.2)$ \\
\hline \multicolumn{5}{|l|}{ Sexo } \\
\hline Masculino & $6.3(1.8)$ & $5.0(2.9)$ & $7.0(2.1)$ & $5.3(1.4)$ \\
\hline Femenino & $6.5(1.7)$ & $4.8(2.8)$ & $6.8(2.2)$ & $5.6(1.0)^{*}$ \\
\hline \multicolumn{5}{|l|}{ Edad } \\
\hline 15 a 17 años & $6.6(1.3)$ & $5.7(2.7)$ & $6.9(1.8)$ & $5.6(0.9)$ \\
\hline 18 a 34 años & $6.2(1.8)$ & $5.2(2.8)$ & $6.5(2.4)$ & $5.4(1.2)$ \\
\hline Arriba de 35 años & $6.3(1.7)$ & $4.5(3.0)$ & $7.2(2.0)^{*}$ & $5.6(1.3)$ \\
\hline \multicolumn{5}{|l|}{ Nivel Educativo } \\
\hline 0-9 años de escolaridad & $6.8(1.4)$ & $5.2(2.8)$ & $7.8(1.6)$ & $5.7(1.0)$ \\
\hline Bachillerato & $6.2(1.7)$ & $4.8(2.9)$ & $6.8(2.0)$ & $5.5(1.2)$ \\
\hline Técnico/Universitario & $5.7(1.9)^{*}$ & $4.6(3.0)$ & $5.8(2.2)^{*}$ & $5.3(1.4)^{*}$ \\
\hline \multicolumn{5}{|l|}{ Estrato social } \\
\hline Alto/medio alto & $5.6(2.0)$ & $4.2(2.9)$ & $5.9(2.5)$ & $5.2(1.4)$ \\
\hline Medio & $6.4(1.6)$ & $5.0(3.0)$ & $6.9(2.0)$ & $5.6(1.2)$ \\
\hline Bajo/Obrero-marginal & $6.5(1.9)^{*}$ & $5.1(2.9)$ & $7.3(2.0)^{*}$ & $5.5(1.2)^{*}$ \\
\hline \multicolumn{5}{|l|}{ Religión } \\
\hline Otra/ninguna & $6.2(2.0)$ & $4.9(3.2)$ & $6.7(2.6)$ & $5.4(1.3)$ \\
\hline Católica & $6.2(1.8)$ & $5.1(2.9)$ & $6.4(2.1)$ & $5.5(1.2)$ \\
\hline Evangélica & $6.5(1.5)$ & $4.6(2.8)$ & $7.6(1.7)^{*}$ & $5.5(1.7)$ \\
\hline
\end{tabular}

Nota. ${ }^{*} p<.05$. Puntajes convertidos a un rango de $0-10$. 
tín-Marín, \& Ramos-Sánchez, 2011; Hernández-Sampieri et al., 2006; John \& Benet-Martínez, 2000).

\section{Resultados}

Con el fin de ofrecer una aproximación al desempeño y los resultados generales de la escala RWA y sus dimensiones, la Tabla 1 sintetiza los promedios obtenidos por la muestra participante atendiendo a variables sociodemográficas consideradas en el cuestionario utilizado.

El promedio de la escala RWA en un rango de 0-10 puntos indica la presencia de un nivel intermedio de actitudes autoritarias en los habitantes del AMSS. Asimismo, las diferencias de medias que muestran significación estadística tanto en la escala general como en sus dimensiones sugieren que el estrato social y el nivel educativo del entrevistado juegan un papel crucial. Es decir que, en la medida en que se cuenta con un nivel técnico o superior de educación, cuestión que suele propender a su vez hacia una movilidad social ascendente, menor nivel de actitudes autoritarias se manifiesta. Por otro lado, a pesar del papel presumiblemente atemperador que el nivel educativo tiene sobre las actitudes autoritarias, el convencionalismo (p. ej., religiosidad más estricta propia de denominaciones evangélicas) parece constituir un caldo de cultivo en el que el RWA encontraría un terreno fértil para su desarrollo. La diferencia significativa entre los puntajes de hombres y mujeres, aunque débil $(p<.03)$ y perteneciente a la dimensión de sumisión autoritaria, puede interpretarse en el mismo sentido.

La confiabilidad de las subescalas del RWA ha sido obtenida a través del Coeficiente Alfa de Cronbach. Las subescalas de agresión autoritaria $(\alpha=.87)$ y la de convencionalismo $(\alpha=$ $.72)$ alcanzaron niveles satisfactorios de consis- tencia interna. La subescala de sumisión autoritaria obtuvo un alfa de Cronbach no satisfactorio ( $\alpha$ $=.49$; ver Taber, 2017). Siguiendo a John y Benet-Martínez (2000), sería esperable que el nivel alfa de escalas nuevas, cortas y cuyo contenido evita ser redundante no cumpla con la convención de lograr un coeficiente alfa superior a .70, el límite inferior que la literatura establece como un nivel alfa adecuado (John \& Benet-Martínez, 2000; Kerlinger \& Lee, 2002; Taber, 2017; Tavakol \& Dennick, 2011). La consistencia interna de una escala, entonces, debe establecerse a partir de su adecuación a los fundamentos teóricos a los que se debe y a su validez, antes que a una mecánica clasificación numérica. Stenner (2005) expone un resultado y una discusión similar a propósito del insatisfactorio nivel alfa de su escala de valores de crianza orientada a medir la predisposición autoritaria.

La validez de constructo fue establecida a través de un análisis factorial confirmatorio (Bordens \& Abott, 1999). La pertinencia del análisis factorial se determinó a partir de los resultados del test de Kaiser-Meyer-Olkin (KMO = .86) y la prueba de esfericidad de Bartlett ( $\chi 2$ [231] = 2049,343; $p<.0001)$. El modelo tridimensional del RWA explica el $40.55 \%$ de la varianza de los resultados y el mismo se puede apreciar en la Figura 1.

Es fácil identificar disensos en la literatura acerca de los índices de bondad de ajuste debido a su diversidad, su alcance o por su sensibilidad al tamaño de la muestra. Esto explica que se reporten varios a la vez y que se coincida con el principio general de Schreiber et al. (2006, p. 327) según el cual "si la vasta mayoría de los índices indica un buen ajuste, entonces probablemente existe un buen ajuste". En la Tabla 2 se muestran los índices de bondad de ajuste obtenidos comparados con los límites ideales que la literatura sugiere. A partir del cotejo de índices de la Tabla 
2 , se puede asumir que el modelo tridimensional del RWA presenta un ajuste satisfactorio.

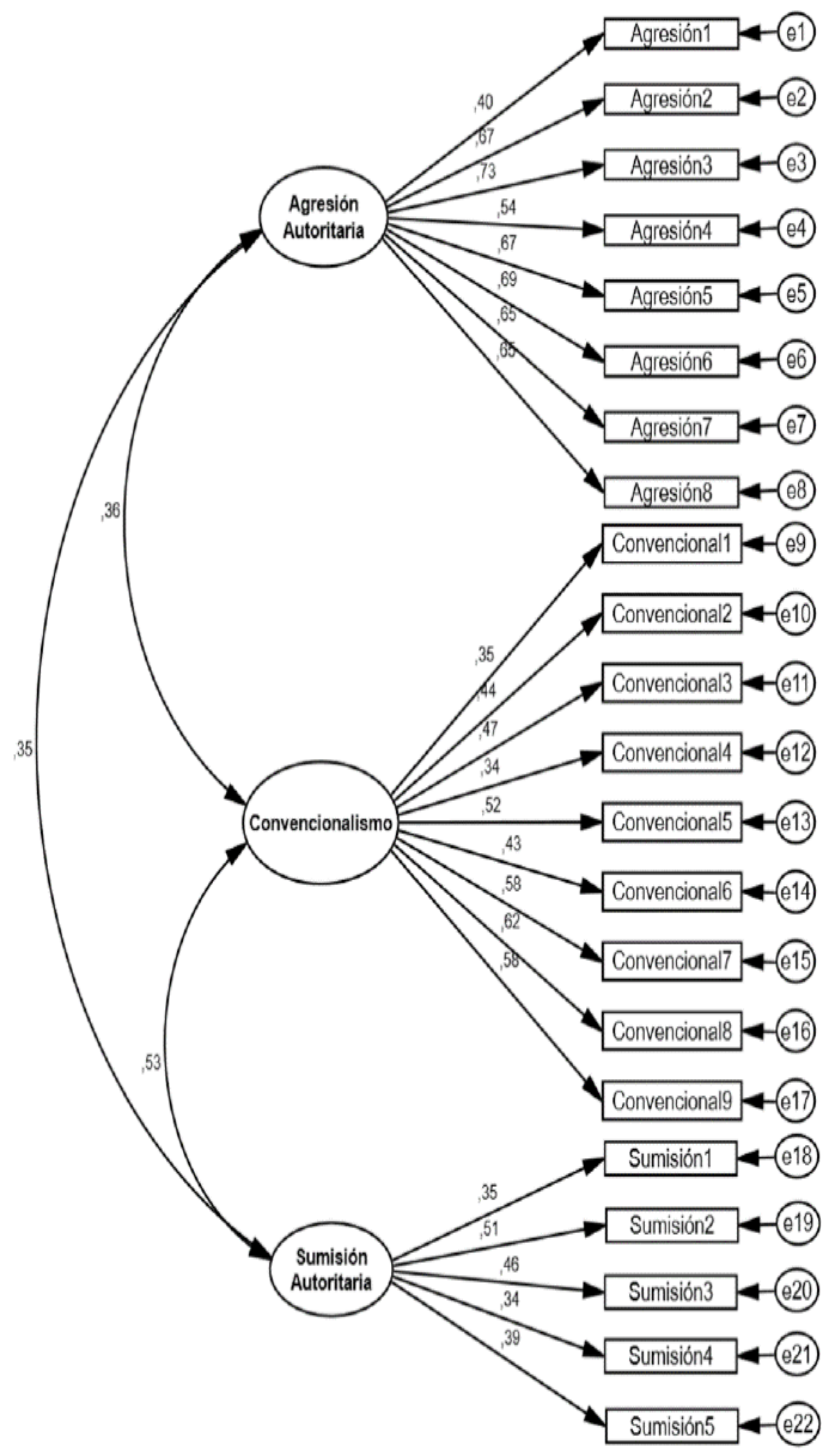

Figura 1

Estructura factorial tridimensional del RWA. Los valores sobre las flechas corresponden a pesos de regresión estandarizados.

Tabla 2

Estadísticos de bondad de ajuste del modelo tridimensional RWA

\begin{tabular}{lcccccc}
\hline & $\boldsymbol{x} \mathbf{2} \boldsymbol{g} \boldsymbol{l}$ & GFI & AGFI & CFI & RMR & RMSEA \\
\hline Obtenidos & 1.86 & .92 & .91 & .90 & .13 & .045 \\
Esperados & $\leq 2.0$ & $>.90$ & $>.90$ & $\geq .95$ & $\begin{array}{c}\text { Cerca } \\
\text { de } 0.0\end{array}$ & .06 \\
& & & & & \\
\hline
\end{tabular}

Nota. Criterios ideales esperados cotejados entre Byrne (2010); Hooper, et al. (2008); Schreiber et al. (2006).
La validez concurrente-de criterio fue establecida a través de correlaciones de Pearson entre la escala RWA y ciertos constructos (criterios) cuya vinculación con el autoritarismo de derechas puede ser hipotetizada a partir de la teoría y la evidencia acumulada (Bordens \& Abott, 1999; Hernández-Sampieri et al., 2006). Como cabría esperar (ver Tabla 3), la escala RWA correlaciona positiva y significativamente con variables de cultura política (p. ej., indiferencia hacia el régimen político/preferencia por un régimen autoritario), de inseguridad ciudadana (p. ej., ha tomado acciones precautorias debido a la delincuencia) y con otras dimensiones psicosociales (p. ej., anomia). En otras palabras, en la lógica del RWA de base de Altemeyer (1996, 2006), la escala RWA que aquí se presenta se vincula con mediciones antidemocráticas, de percepción de amenaza, de punitividad y concepciones peyorativas de otros grupos (p. ej., prejuicio hacia delincuentes: considerar que los delincuentes son dementes o que nacen como tales).

\section{Discusión}

La escala presentada, por su nombre, no debe conducir a equívocos. Su denominación-Escala Salvadoreña de Autoritarismo de Derechasno remite a imperativo alguno de administración exclusiva en El Salvador. Más bien significa que responde a realidades similares a la salvadoreña. Como fue dicho, son sociedades que experimentan déficits democráticos, desigualdad y exclusión, altos niveles de violencia e inseguridad así como culturas conservadoras y tradicionalistas, entre otros aspectos. Características como estas son identificables en buena parte de Latinoamérica. Asimismo, la escala ostenta un origen realista al surgir de ítems probados y depurados en encuestas de opinión y a partir de muestras reales 
Tabla 3

Correlaciones relevantes entre las subescalas del RWA y variables de interés teórico-empírico.
(1)
(2)
(3)
(4) (5)
(6)
(7)
(8)
(9) (10)
(11)
(12)

(1) Agresión autoritaria

(2) Convencionalismo

$.36^{* *}$

(3) Sumisión autoritaria

$.35^{* *} .53 * *$

(4) Ideología política ha- $.00 \quad .24^{* *} .26^{* *}$ cia la derecha

(5) Indiferente/prefiere ré- $\quad .19^{* *} \quad .10 \quad .08 \quad .06$ gimen autoritario



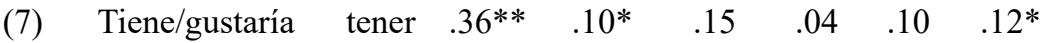
arma de fuego ma en barrio

(9) Vio una persona asesi- $\begin{array}{lllllllll}.19 * * & .00 & -.01 & .02 & .04 & .32 * * & .10^{*} & .26 * *\end{array}$ nada en la calle

$\begin{array}{llllllllll}\text { (10) Disposición autoritaria } & .18^{* *} & .48 * * & .37 * * & .08 & .07 & .01 & .02 & .03 & .01\end{array}$

(11) Anomia $\quad .34 * * 32 * * \begin{array}{lllllllll} & .42 * & -.03 & .10^{*} & .14 * * & .17 * * & .18 * * & .13 * & .05\end{array}$

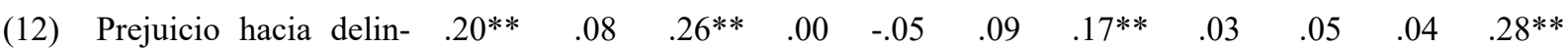
cuentes

Nota. ${ }^{*} p<.05, * * p<.01$. Las variables incluidas en la encuesta administrada que aquí se correlacionan con las subescalas del RWA fueron codificadas así: Ideología política de derecha es una escala de 10 puntos $(1=$ izquierda, $10=$ derecha); indiferente/prefiere régimen autoritario es una variable ficticia (dummy; 1 = indiferente o prefiere régimen autoritario, $0=$ prefiere régimen democrático); delincuencia ha empeorado es una variable ficticia $(1=$ delincuencia ha empeorado, $0=$ delincuencia sigue igual/ha disminuido); acciones precautorias corresponde a una escala de $0-10$ puntos (puntajes tendientes a 10 indican más acciones precautorias tomadas); tiene/gustaría tener un arma de fuego es una variable ficticia ( 1 = Tiene/gustaría tener un arma de fuego, $0=$ no tiene/ni gustaría tener un arma de fuego); pandillas como problema en el barrio corresponde a una escala de 0-3 puntos (puntajes tendientes a 3 refieren mucho problema); vio persona asesinada en la calle es una pregunta dicotómica $(1=$ sí, $2=$ no); disposición autoritaria es una escala que va de 0-10 puntos $(0=$ libertarismo, $10=$ autoritarismo); anomia corresponde a una escala de 0-10 puntos (puntajes tendientes a 10 indican percepción de ano $^{\text {mia); }}$; prejuicio hacia delincuentes corresponde a una escala de 0-10 puntos (puntajes tendientes a 10 indican mayor prejuicio).

y representativas en términos teóricos y metodológicos. Casi sin excepción, los estudios disponibles sobre autoritarismo que emplean alguna variante de la escala RWA de Altemeyer (1996) $\mathrm{u}$ otra alternativa de medición trabajan con muestras socialmente irrelevantes (i.e., estudiantes universitarios).

Precisamente esta lealtad al contexto es lo que puede explicar y justificar la dificultad de alcanzar límites ideales de ciertos índices o el margen de mejora de la escala. No faltará quien sugiera la fusión de dimensiones o el retiro de ítems para seguir incrementando coeficientes y bondades de ajuste. Pero, por ejemplo, la dimensión de sumisión autoritaria tiene una "identidad teórica propia" suficientemente distintiva de la de convencionalismo y métricamente la fusión de ambas escalas no conlleva mejoras a la propuesta tridimensional (i.e., no se reducen sus ítems o empeoran los indicadores de bondad de ajuste del modelo). No obstante, es claro que el bajo nivel de consistencia interna de la subescala de sumisión autoritaria plantea la necesidad de realizar esfuerzos adicionales de medición. A su vez, el ítem 1 y el ítem 4 de la escala de convencionalismo, los que presentan los pesos de regresión más bajos de la dimensión (ver Figura 1: ítem $1=.35$, ítem 4 $=.34$ ) y que usualmente suelen ser elegibles para 
ser depurados, respectivamente, remiten a aspectos contextuales relevantes como son el clamor popular porque el ejército participe en labores de seguridad y la reconocida homofobia de la cultura salvadoreña.

La medición mecánica y abstraída del contexto de sentido donde aquella tiene lugar puede conducir a un ejercicio tecnocrático vacío. De esta manera, la escala cumple con un cometido técnico pero también social sin el cual lo primero pierde relevancia. Los análisis realizados han establecido que existen indicios métricos suficientes para considerar la escala salvadoreña RWA como confiable y válida para la medición de actitudes autoritarias (Altemeyer, 2006) en contextos como el salvadoreño, al alcanzar con éxito la mayoría de guarismos establecidos por la literatura especializada disponible (Bordens \& Abott, 1999; Byrne, 2010; Cubo-Delgado et al., 2011; Hernández-Sampieri et al., 2006; Hooper et al., 2008; John \& Benet-Martínez, 2000; Schreiber et al., 2006).

El nivel de actitudes autoritarias obtenido con la escala $(\mathrm{M}=6.3 ; \mathrm{DE}=1.7)$, converge con el reportado en otros estudios. Se destaca también su sensibilidad ante características de desventaja social de los participantes, para las que el nivel de estudio resulta ser un modulador importante (Córdova-Macías et al., 2015; Cruz, 1999; Cruz et al., 2017; IUDOP, 2002). Según Córdova-Macías et al., (2015), en "las Américas", los años de educación constituyen el factor de mayor peso explicativo de la tolerancia política $y$, entre otras variables, la mujeres tienden mostrar menor tolerancia política que los hombres. La recurrente relación inversa que se encuentra entre nivel educativo $\mathrm{y}$ autoritarismo en encuestas de opinión en las que participa población salvadoreña (y latinoamericana en general) sugiere que el autoritarismo que es detectado en estas latitudes responde, prioritariamente, a las tendencias prejuiciosas que mezclan baja habilidad cognitiva, sensibilidad a la amenaza y respuesta conservadora o característica de ala derecha dirigida a grupos considerados como no convencionales, como serían personas ateas o pertenecientes a la comunidad LGTBI (Brandt \& Crawford, 2016).

Las correlaciones bivariadas son consistentes con la lógica teórica del RWA de Altemeyer al asociarse con percepciones varias de amenaza que confirman el mundo como un lugar peligroso ( $\mathrm{p}$. ej., pretensión de armarse, tomar acciones contra el crimen, creer que la delincuencia ha empeorado) o que alegan roto el consenso y la estabilidad del entorno, lo que incluye el orden democrático (p. ej., preferir un régimen autoritario, anomia; Feldman \& Stenner, 1997; Merolla \& Zechmeister, 2009; Sibley et al., 2007; Stenner, 2005). La suficiencia del modelo, comprobado a través del análisis factorial confirmatorio y corroborado con los indicadores de bondad de ajuste, avala la propuesta tridimensional de la escala RWA. Al mismo tiempo, estos resultados apoyan la robustez teórica que subyace al RWA de Altemeyer (1981, 2006) y su resistencia al paso del tiempo y a las adaptaciones y variaciones de que ha sido objeto.

Aunque este trabajo sortea la usual limitación y/o recomendación de buscar una muestra representativa como paso siguiente y que el modelo tridimensional de la escala salvadoreña RWA ha probado con suficiencia ser apropiado y sus escalas confiables y válidas, es posible recomendar la exploración de vinculaciones clásicas en el estudio del autoritarismo. Las covariaciones con constructos como anomia, prejuicio y disposición autoritaria (ver Tabla 3) son indicios teórico-metodológicos que deben consolidarse con otras exploraciones que incluyan constructos como la orientación de dominancia social o características de personalidad como The Big Five (John, Naumann \& Soto, 2008). En este cometido sería interesante además poner a prueba la relación entre la 
escala aquí presentada y la misma escala RWA de Altemeyer (1996) o alguna de sus variantes disponibles en español.

\section{Referencias}

Adorno, T. W., Frenkel-Brunswik, E., Levinson, D. J., \& Sanford, R. N. (1969). The Authoritarian Personality. New York: The Norton Library.

Allport, G. W. (1954). La naturaleza del prejuicio. Buenos Aires: EUDEBA.

Altemeyer, B. (1981). Right-Wing Authoritarianism. Winnipeg: University of Manitoba Press.

Altemeyer, B. (1996). The Authoritarian Specter. Cambridge: Harvard University Press.

Altemeyer, B. (1998). The other "authoritarian personality". Advances in Experimental Social Psychology, 30, 47-92. doi: 10.1016/S0065-2601(08)60382-2

Altemeyer, B. (2006). The Authoritarians. Recuperado de https://theauthoritarians.org/

Arbuckle, J. L. (2012). IBM ${ }^{\circledR} S P S S ® \operatorname{Amos}^{\mathrm{TM}} 21$ User's $^{\prime}$ Guide. Recuperado de http://public.dhe.ibm.com/ software/analytics/spss/documentation/amos/21.0/ en/Manuals/IBM_SPSS_Amos_Users_Guide.pdf

Bordens, K. S., \& Abott, B. B. (1999). Research Design and Methods. A Process Approach (4 ${ }^{\mathrm{a}} \mathrm{ed}$.). Mountain View, CA: Mayfield Publishing Company.

Brandt, M. J., \& Crawford, J. T. (2016). Answering unresolved questions about the relationship between cognitive ability and prejudice. Social Psychological and Personality Science, 7(8), 884-892. doi: 10.1177/1948550616660592

Byrne, B. M. (2010). Structural equation modeling with AMOS. Basic concepts, applications, and programming ( $2^{\mathrm{a}}$ ed.). New York, NY: Routledge.

Canetti, D., \& Pedahzur, A. (2002). The effects of Contextual and Psychological Variables on Extreme Right-wing Sentiments. Social Behavior and Personality: An International Journal, 30(4), 317-334. doi: 10.2224/sbp.2002.30.4.317
Córdova-Macías, R., Cruz, J. M., \& Zeichmeister, E. (2015). Cultura política de la democracia en El Salvador y en las Américas, 2014: Gobernabilidad democrática a través de 10 años del Barómetro de las Américas. San Salvador: FUNDAUNGO y LAPOP-Universidad de Vanderbilt. Recuperado de https://www.vanderbilt.edu/lapop/el salvador.php

Cruz, J. M. (1999). El autoritarismo en la posguerra: un estudio de las actitudes de los salvadoreños. Estudios Centroamericanos (ECA), (603), 95-106.

Cruz, J. M., Aguilar, J., \& Vorobyeva, Y. (2017). Legitimidad y confianza pública de la policía en El Salvador. San Salvador: KG-LACC, FIU \& IUDOP.

Cubo-Delgado, S., Martín-Marín, B., \& Ramos-Sánchez, J. L. (Coords.). (2011). Métodos de investigación y análisis de datos en Ciencias Sociales y de la Salud. Madrid: Pirámide.

Dirección General de Estadística y Censos (2009). República de El Salvador. Estimaciones y proyecciones de la población 1950-2050. San Salvador: MINEC, UNFPA \& CELADE. Recuperado de http://www. censos.gob.sv

Duckitt, J. (1992). Psychology and prejudice: A historical analysis and integrative framework. American Psychologist, 47(10), 1182-1193. doi: 10.1037/0003066X.47.10.1182

Duckitt, J. (2001). A dual-process cognitive-motivational theory of ideology and prejudice. En M. P. Zanna (Ed.), Advances in Experimental Social Psychology (Vol. 33, pp. 41-113). San Diego, CA: Academic Press.

Duckitt, J. (2003). Prejudice and intergroup hostility. En D. Sears, L. Huddy, \& R. Jervis (Eds.), Oxford Handbook of Political Psychology (pp. 559-600). New York, NY: Oxford University Press.

Etchezahar, E. (2012). Las dimensiones del autoritarismo: Análisis de la escala de autoritarismo del ala de derechas (RWA) en una muestra de estudiantes universitarios de la Ciudad de Buenos Aires. Psicología Política, 12(25), 591-603. Recuperado de http://www. psicologiapolitica.org 
Feldman, S., \& Stenner, K. (1997). Perceived threat and authoritarianism. Political Psychology, 18(4), 741-770. doi: 10.1111/0162-895x.00077

Fernández, A., Pérez, E., Alderete, A. M., Richaud, M. C., \& Fernández-Liporace, M. (2010). ¿Construir o adaptar tests psicológicos? Diferentes respuestas a una cuestión controvertida. Revista Evaluar, 10(1), 60-74. Recuperado de https://revistas.unc.edu.ar/index.php/revaluar

Fournier, M., \& Garnier, L. (1990). Fórmula abreviada para la transformación de puntajes de variables a una misma escala. Revista Latinoamericana de Psicología, 22(3), 421-426. Recuperado de http://www.redalyc. org/revista.oa? $\mathrm{id}=805$

Giampaglia, G., \& Roccato, M. (2002). La scala di autoritarismo di destra di Altemeyer: Un'analisi con il modello di Rasch per la costruzione di una versione italiana. Testing, psicometria e metodologia, 9, 93111. Recuperado de http://hdl.handle.net/2318/8897

Hernández-Sampieri, R., Fernández-Collado, C., \& Baptista Lucio, P. (2006). Metodología de la Investigación (4 ${ }^{\mathrm{a}}$ ed.). México: McGraw-Hill Interamericana.

Hooper, D., Coughlan, J., \& Mullen, M. (2008). Structural ecuation modelling: Guidelines for determining model fit. Electronic Journal of Business Research Methods, 6(1), 53-60. Recuperado de http://www.ejbrm. com/main.html

Instituto Universitario de Opinión Pública. (1998). Encuesta de evaluación de Derechos Humanos (IUDOP Informe 67). Recuperado de http://www.uca.edu.sv/ iudop/?page_id=11

Instituto Universitario de Opinión Pública(1999a). Encuesta sobre valores (IUDOP Informe 80). Recuperado de http://www.uca.edu.sv/iudop/?page_id=11

Instituto Universitario de Opinión Pública (1999b). Normas culturales y actitudes sobre la violencia - Estudio Activa. San Salvador: Autor.

Instituto Universitario de Opinión Pública (2002). Encuesta sobre actitudes autoritarias en jóvenes residentes en la zona urbana del municipio de San Salvador (IUDOP Informe 96). Recuperado de http://www.uca. edu.sv/iudop/?page_id=11

Instituto Universitario de Opinión Pública (2014). La situación de la seguridad y de la justicia 2009-2014. Entre expectativas de cambio, mano dura militar $y$ treguas. San Salvador: Autor. Recuperado de http:// www.uca.edu.sv/iudop/?page_id=31

John, O. P., \& Benet-Martínez, V. (2000). Measurement: Reliability, construct validation, and scale construction. En H. T. Reis y C. M. Judd (Eds.), Handbook of Research Methods in Social and Personality Psychology (pp. 339-369). Cambridge: Cambridge University Press.

John, O. P., Naumann, L. P., \& Soto, C. J. (2008). Paradigm shift to the integrative Big-Five trait taxonomy: History, measurement, and conceptual issues. En O. John, R. Robins \& L. Pervin (Eds.), Handbook of Personality: Theory and Research (pp. 114-158). New York: Guilford Press.

Kerlinger, F. N., \& Lee, H. B. (2002). Investigación del Comportamiento. Métodos de Investigación en Ciencias Sociales (4⿳a ed.). México: McGraw-Hill Interamericana.

Latinobarómetro (2015). Informe Latinobarómetro 2015: Veinte años de opinión pública 1995-2015. Recuperado de http://www.latinobarometro.org

McClosky, H., \& Schaar, J. H. (1965). Psychological dimensions of anomy. American Sociological Review, 30(1), 14-40. doi: 10.2307/2091771

Merolla, J. L., \& Zechmeister, E. J. (2009). Democracy at Risk. How terrorist Threats Affect the Public. Chicago, IL.: The University of Chicago Press.

Molero, F. (2007). El estudio del prejuicio en la psicología social. En J. Morales, M. Moya, E. Gaviria \& I. Cuadrado (Eds.), Psicología Social (3ª ed., pp. 591-617). Madrid: McGraw Hill.

Moya, M., \& Morales-Marente, E. (2005). Reacciones psico-políticas ante los ataques terroristas del $11 \mathrm{de}$ Marzo de 2004. Revista de Psicología Social, 20(3), 331-350. doi: 10.1174/021347405774277749

Orellana, C. I. (2012). Exclusión, crisis del mundo del trabajo y precariedad. A vueltas con el tema de la ciuda- 
danía. Estudios Centroamericanos (ECA), 67(729), 229-258.

Orellana, C. I. (2017). Proclivity to hate: Violence, group targeting and authoritarianism in El Salvador. En E. Dumbar, A. Blanco, \& D. Crévecoeur-MacPhail (Eds.), The Psychology of Hate Crimes as Domestic Terrorism: U.S. and Global Issues (Vol. 1, pp. 239281). Santa Barbara, CA.: Praeger Publishers.

Ovejero, A. (1992). Sobre la cuestión del autoritarismo de izquierdas. Psicología Política, 5, 53-69. Recuperado de https://www.uv.es/garzon/psicologia\%20politica/index.html

Programa de las Naciones Unidas para el Desarrollo (2013). Informe sobre Desarrollo humano El Salvador 2013. Imaginar un nuevo país. Hacerlo posible. San Salvador: Autor. Recuperado de http://www.sv.undp.org/ content/el_salvador/es/home.html

Roccato, M., \& Converso, D. (1996). Cómo y porqué es necesario volver a estudiar el autoritarismo. Psicología Política, 13, 63-79. Recuperado de https://www. uv.es/garzon/psicologia\%20politica/index.html

Rokeach, M. (1960). The open and closed mind. Investigations into the nature of belief systems and personality systems. New York, NY: Basic Books.

Schreiber, J. B., Nora, A., Stage, F. K., Barlow, E. A., \& King, J. (2006). Reporting structural equation modeling and confirmatory factor analysis results: A review. The Journal of Educational Research, 99(6), 323-337. doi: 10.3200/joer.99.6.323-338

Seligson, M. A., Cruz, J. M., \& Córdova-Macías, R. (2000). Auditoría de la democracia. El Salvador 1999. San Salvador: FUNDAUNGO, IUDOP \& Universidad de Pittsburgh. Recuperado de http://fundaungo.org. SV

Seoane, J., \& Garzón, A. (1992). Creencias sociales contemporáneas, autoritarismo y humanismo. Psicología Política, 5, 27-52. Recuperado de https://www. uv.es/garzon/psicologia\%20politica/index.html https://www.uv.es/garzon/psicologia\%20politica/ N5-2.pdf

Sibley, C., Wilson, M., \& Duckitt, J. (2007). Effects of dan- gerous and competitive worldviews on right-wing authoritarianism and social dominance orientation over a five-month period. Political Psychology, 28(3), 357-371. doi: 10.1111/j.1467-9221.2007.00572.x

Speizer, I., Goodwin, M., Samandari, G., Kim, S., \& Clyde, M. (2008). Dimensions of child punishment in two Central American countries: Guatemala and El Salvador. Revista Panamericana de Salud Pública, 23(4), 247-256. doi: 10.1590/s1020-49892008000400004

Srole, L. (1956). Social integration and certain corollaries: An exploratory study. American Sociological Review, 21(6), 709-716. doi: 10.2307/2088422

Stenner, K. (2005). The Authoritarian Dynamic. New York: Cambridge University Press.

Stone, W. (1990). Autoritarismo de izquierdas: Aún sin demostrar. Psicología Política, 1, 13-34. Recuperado de https://www.uv.es/garzon/psicologia\%20politica/ index.html

Taber, K. S. (2017). The use of Cronbach's alpha when developing and reporting research instruments in science education. Research in Science Education, 1-24. doi: 10.1007/s11165-016-9602-2

Tavakol, M., \& Dennick R. (2011). Making sense of Cronbach's alpha. International Journal of Medical Education, 2, 53-55. doi: 10.5116/ijme.4dfb.8dfd

Van Hiel, A., Duriez, B., \& Kossowska, M. (2006). The presence of left-wing authoritarianism in western europe and its relationship with conservative ideology. Political Psychology, 27(5), 769-793. doi: 10.1111/j.1467-9221.2006.00532.x

Zakrisson, I. (2005). Construction of a short version of the Right Wing Authoritarianism (RWA) scale. Personality and Individual Differences, 39(5), 863-872. doi: 10.1016/j.paid.2005.02.026 
Apéndice 1

Escala salvadoreña de autoritarismo de derechas (RWA) separada por dimensiones

Instrucciones generales: Podría indicarme qué tan de acuerdo o en desacuerdo se encuentra con las siguientes afirmaciones/situaciones...

(4) Muy de acuerdo, (3) Algo de acuerdo, (2) Indeciso, (1) Algo en desacuerdo, (0) Muy en desacuerdo

Agresión autoritaria
1. Un grupo de personas comience a matar a gente que consideran indeseable

2.Si las autoridades fallan, la gente tiene derecho de tomar la justicia por su propia cuenta

3. La única forma de sacar adelante a nuestro país es eliminar por la fuerza a los que causen problemas

4.En algunos casos, se justifica que la policía torture a los sospechosos para obtener información

5.En su comunidad o colonia capturen a un delincuente reconocido y lo linchen entre todos

6.En algunas ocasiones, la policía debería matar a los delincuentes en lugar de intentar apresarlos

7.Se deberían eliminar a los pandilleros para acabar con los constantes pleitos entre ellos

8.Se debería de implantar la pena de muerte en el país

\section{Convencionalismo}

1.La presencia militar en las calles es necesaria para controlar la delincuencia en el país

2.Para que una familia pueda funcionar bien debe estar compuesta por un padre, una madre e hijos/as

3.Las mujeres deben ser las encargadas de las tareas domésticas

4.La homosexualidad es una perversión

5.Los problemas que tienen las personas en el país se dan porque se han alejado de dios

6.Los desastres naturales son un castigo divino

7.Todos los problemas que están sucediendo en el mundo en la actualidad están escritos en la Biblia

8.El principal objetivo en la vida es fundar una familia

9.El hombre debe ser la cabeza de la familia

\section{Sumisión autoritaria}

1.Es mejor vivir en una sociedad ordenada, a pesar de que con ello se limiten algunas libertades

2.En lugar de derechos humanos, lo que nuestro país necesita en realidad es mucha ley y orden

3.Una buena educación escolar es aquella que enfatiza la disciplina y la obediencia de los alumnos

4.Aquellos que critican la forma en que está organizada la sociedad son resentidos sociales inconformes

5.El amor a la nación se demuestra rechazando a aquellos que critican a sus autoridades 